\title{
Factores asociados al abandono de terapia antirretroviral de alta efectividad en pacientes con VIH SIDA en un hospital de tercer nivel
}

\author{
Factors associated with the abandonment \\ of highly effective antiretroviral therapy in \\ patients with HIV in a third-level hospital
}

\author{
Ana María Granada, Claudia Vanegas, Elizabeth Forero, \\ Claudia Silva, Erika Paola Vergara • Bogotá, D.C. (Colombia)
}

\section{Resumen}

Introducción: la baja adherencia en el tratamiento antirretroviral de alta efectividad TARGA se asocia a peores desenlaces en salud; además de las comorbilidades infecciosas, muchos pacientes con VIH/SIDA presentan asociado psicopatología, farmacodependencia y problemas socioeconómicos. Existe alta frecuencia de abandono a la terapia en nuestro medio.

Objetivo: identificar las variables asociadas al abandono de terapia TARGA en pacientes con VIH de un hospital de tercer nivel en Colombia.

Métodos y resultados estudio descriptivo corte transversal: se incluyeron 51 pacientes pertenecientes al programa durante un año; 12 pacientes (23\%) tuvieron adherencia al tratamiento, 32 (62\%) tenían antecedente de consumo de sustancias psicoactivas y $33(64 \%)$ tenían asociada alguna patología psiquiátrica. Los factores que se asociaron a la no adherencia fueron la psicopatología y la pobre red de apoyo.

Conclusión: En la población a estudio se documenta alta tasa de abandono a la terapia TARGA, probablemente asociado a enfermedad mental y pobre red social. (Acta Med Colomb 2018; 43: 31-36).

Palabras clave: SIDA, VIH, terapia antirretroviral de alta efectividad, adherencia a medicación, enfermedad mental.

\footnotetext{
Abstract

Introduction: the low adherence in the highly effective antiretroviral treatment HAART is associated with worse health outcomes. In addition to infectious comorbidities, many patients with HIV / AIDS have associated psychopathology, drug dependence and socioeconomic problems. There is a high frequency of abandonment of therapy in our environment.

Objective: to identify the variables associated with the abandonment of HAART therapy in patients with HIV from a third level hospital in Colombia.

Methods and Results: descriptive cross-sectional study: 51 patients belonging to the program during one year were included; 12 patients (23\%) had adherence to treatment, 32 (62\%) had a history of psychoactive substance use and $33(64 \%)$ had some psychiatric pathology. The factors that were associated with non-adherence were psychopathology and the poor support network.

Conclusion: high rate of abandonment of HAART therapy is documented in the study population, probably associated with mental illness and poor social network. (Acta Med Colomb 2018; 43: 31-36).

Keywords: AIDS, HIV, highly effective antiretroviral therapy, adherence to medication, mental illness.
}

Dra. Ana María Granada Copete: Internista. Coordinadora Programa VIH, Hospital Santa Clara; Dra. Claudia Vanegas: Psiquiatra Hospital Santa Clara. Docente Psiquiatría Universidad El Bosque; Elizabeth Forero Rozo: Enfermera. Programa VIH Hospital Santa Clara; Claudia Silva: Terapeuta Ocupacional. Programa VIH Hospital Santa Clara, Dra. Erika Paola Vergara: Internista. Epidemióloga Hospital Santa Clara. Bogotá, D.C. (Colombia).

Correspondencia: Dra. Erika Paola Vergara. Bogotá, D.C. (Colombia).

E-mail: paoli1982@gmail.com

Recibido: 21/IX/2016 Aceptado: 12/II/2018 


\section{Introducción}

El advenimiento de los medicamentos antirretrovirales generó un antes y un después en la epidemia del síndrome de inmunodeficiencia adquirida (SIDA), transformando la entidad catalogada como mortal a una enfermedad crónica, lo que ha generado una alta expectativa frente a la morbilidad asociada, disminución de nuevos casos y por ende un aumento en los años de vida saludable. Para alcanzar estos logros se requieren esfuerzos multidisciplinarios e interinstitucionales que permitan impactar el curso de la enfermedad, junto a la oferta tanto de servicios de salud oportunos como de otras dependencias que tienen que ver con condiciones socioeconómicas y culturales que intervienen en el cuidado de la salud.

Se ha reconocido a nivel mundial la diversidad en la población con diagnóstico de VIH/Sida, la importancia de ofertar diagnóstico temprano así como tratamiento integral y existen dificultades en ambos casos dadas por determinantes sociales (ser trabajador sexual, tener relaciones sexuales hombre con hombre, usar drogas psicoactivas) y otros factores de vulnerabilidad. Para Colombia la pobreza, las migraciones, los desplazamientos forzados, el conflicto armado y el estigma en personas que viven con el VIH, dificultan la oportunidad diagnóstica, el seguimiento y tratamiento.

La implementación de guías de atención a la población adolescente, adultos, niños y mujeres en edad reproductiva para diagnostico y tratamiento del VIH/Sida (1) busca mejorar la oportunidad diagnostica y terapéutica; sin embargo, persisten dificultades que traen resultados poco favorables en el impacto de la atención y contención de la epidemia. El gran reto es mejorar la adherencia, numerosas personas que comienzan el tratamiento del SIDA no se vinculan adecuadamente a los servicios sanitarios, abandonan el tratamiento y regresan en una fase demasiado tardía, cuando la terapia antirretroviral ha perdido eficacia. En Estado Unidos según registros de la Organización de Naciones Unidas (ONU) SIDA 18\% de los pacientes no conocen su diagnóstico, $66 \%$ están vinculados a la atención en salud, y sólo $37 \%$ de los pacientes atendidos en programa son adherentes; el 25\% tienen carga viral suprimida. En nueve países de África subsahariana, el recuento promedio de linfocitos TCD4 al iniciar el tratamiento del VIH en 2010 era inferior al umbral crítico, que pone en peligro la vida, éste es de 200 células $/ \mathrm{mm}^{3}$, cifra sustancialmente menor a la establecida en las normas recomendadas para el inicio óptimo del tratamiento (2).

En Colombia a través de los indicadores de alerta temprana, promovidos por la Organización Mundial de la Salud (OMS) y liderados por el Instituto Nacional de Salud, INS (3) se realiza seguimiento para determinar: prácticas de prescripción de antirretrovirales (ARV), reclamo puntual de los medicamentos, asistencia puntual a las consultas, porcentaje de pacientes con pérdida de seguimiento y porcentaje de pacientes que continúan recibiendo tratamiento al cabo de 12 meses; también se hace vigilancia por desabastecimiento y escasez de estos medicamentos.
La no adherencia a los antirretrovirales se asocia a resistencia a los medicamentos y a primo infección con virus resistentes, impactando en forma negativa el control de la epidemia (4-7).

En nuestra institución (tercer nivel que atiende población vulnerable en servicios ambulatorios y de hospitalización) se percibe un diagnostico tardío de la entidad, los pacientes presentan estados avanzados y debutan con enfermedades marcadores como tuberculosis y criptococosis, lo que empobrece su proceso de recuperación e impacta sobre su expectativa y calidad de vida; por otra parte se suman determinantes sociales y psicológicas como el consumo de sustancias psicoactivas y la pobre red de apoyo. Para el año 2012, dentro del estudio de AIT (alerta temprana a resistencia a antirretrovirales del Instituto Nacional de Salud) se encontró que $90 \%$ de los pacientes pertenecientes al programa habían abandonado la terapia durante el primer año de tratamiento, $40 \%$ reclamo oportunamente medicamentos y sólo $22,7 \%$ acudieron a cita de control. Como es de esperarse se logró carga viral suprimida a los 12 meses sólo en $15 \%$ de la población (3).

La identificación de variables probablemente relacionados con el abandono de la terapia se hace fundamental en términos de posibles intervenciones de las mismas; considerando lo anterior el objetivo del trabajo es identificar factores asociados a la no adherencia y abandono de la terapia antirretroviral en pacientes adultos con VIH atendidos en un hospital de tercer nivel. Como hipótesis de trabajo se plantea que la no adherencia está asociada a variables psicopatológicas más que a barreras de acceso a tratamiento.

\section{Material y métodos}

Estudio descriptivo prospectivo de corte transversal. Durante un año (junio 2013 a junio 2014), previa firma de consentimiento informado, se obtuvieron datos de pacientes adultos del programa institucional de VIH/Sida vistos de forma ambulatoria y hospitalizada que recibieran terapia antirretroviral. El protocolo fue aprobado por los comités de investigación de la institución. Se incluyó la totalidad de la población en el periodo del estudio. Se recolectaron datos sociodemográficos y del estado de salud (coinfecciones y comorbilidades asociadas o no a VIH/Sida). El estado inmunológico se evaluó con conteo de células T CD4 y la carga viral para VIH -1. En la valoración de los trastornos psicopatológicos se realizaron: entrevistas estructuradas para la detección de psicopatología (Mini-International Neuropsiquiatric Interview) $(8,9)$ y entrevistas para la detección de trastornos de Personalidad del Eje II del DSM IV (SCID II cuestionario de personalidad) (10). Como pruebas de tamizaje de trastorno neuro-cognitivo se utilizó el MINI Mental test (11-13) y la escala MOCA (14). La prueba de tamizaje de trastorno neurocognitivo específicas para SIDA usada fue la Escala de Demencia para Sida (Internacional HIV Dementia Scale). (15-17). 
La adherencia se valoró con la escala de Sacket, en la que se tiene en cuenta total de pastillas tomadas, porcentaje de cumplimiento a citas de seguimiento (mayor de $80 \%$ ) y porcentaje de reclamos del medicamento en farmacia mayor al $80 \%$. Además en forma indirecta se consideró un paciente adherente cuando presentaba mejoría en conteo CD4 y carga viral indetectable (18).

Las variables numéricas continuas se midieron con promedios y desviaciones estándar, y las variables categóricas como frecuencias. Se realizó análisis univariado, utilizando diferencias de proporciones y el test chi-cuadrado para variables categóricas; se tomarán como estadísticamente significativas aquellas con valor de $\mathrm{p}$ menor a 0.05 . Se realizó regresión logística con las variables significativas. Se utilizaron los programas estadísticos EXCEL y SPSS versión 23 IBM.

Tabla 1. Carácterísticas de la población.

\begin{tabular}{|c|c|}
\hline & $\mathrm{N}=51$ \\
\hline \multicolumn{2}{|l|}{$\operatorname{Sexo} \mathbf{n}(\%)$} \\
\hline Hombre & $46(90 \%)$ \\
\hline \multicolumn{2}{|l|}{ Género n(\%) } \\
\hline Heterosexual & $25(49 \%)$ \\
\hline Homosexual & $21(41 \%)$ \\
\hline Bisexual & $4(7.8 \%)$ \\
\hline Transgénero & $1(2 \%)$ \\
\hline Diagnóstico mayor a 2 años $n(\%)$ & $28(55 \%)$ \\
\hline Edad promedio (rango) & 41.2 (24-60 años) \\
\hline Algún tipo de empleo n(\%) & $39(76 \%)$ \\
\hline \multicolumn{2}{|l|}{ Tipo de vivienda $\mathbf{n}(\%)$} \\
\hline Propia & $17(33 \%)$ \\
\hline Arriendo & $30(58 \%)$ \\
\hline Habitante de la calle & $2(3.9 \%)$ \\
\hline Otros & $2(3.9 \%)$ \\
\hline \multicolumn{2}{|l|}{ Ingresos mensuales $\mathbf{n}(\%)$} \\
\hline Entre 0 a 2 SMLV & $48(94 \%)$ \\
\hline Mayor a 2 SMLV & $3(5.9 \%)$ \\
\hline \multicolumn{2}{|l|}{ Estrato socioeconómico n(\%) } \\
\hline 0 & $3(5.9 \%)$ \\
\hline 1 & $9(17.6 \%)$ \\
\hline 2 & $24(47 \%)$ \\
\hline 3 & $15(29 \%)$ \\
\hline \multicolumn{2}{|l|}{ Red de apoyo n(\%) } \\
\hline Sin red & $3(5.9 \%)$ \\
\hline Con red & $48(94 \%)$ \\
\hline Consumo sustancias psicoactivas $\mathbf{n}(\%)$ & $32(62 \%)$ \\
\hline
\end{tabular}

\section{Resultados}

Durante el periodo de estudio se incluyeron 51 pacientes, $46(90 \%)$ eran hombres. Del total (41\%) homosexuales, 28 pacientes $(55 \%)$ tenían diagnóstico mayor a dos años. El promedio de edad fue 41 años (rango de 24-60). 38 personas $(74 \%)$ eran solteros y $12(23 \%)$ eran desempleados. 24 pacientes $(47 \%)$ pertenecían a estrato 2 , el segundo más frecuente estrato 1 ( 9 pacientes). 48 pacientes contaban con red de apoyo, 32 pacientes (62\%) habían consumido sustancias psicoactivas en algún momento de la vida, de ellos 10 no habían suspendido el consumo en los últimos dos años; tres pacientes fallecieron durante el periodo de estudio (Tabla 1).

En la Tabla 2 se resumen las variables clínicas. La tuberculosis fue la infección oportunista más frecuente (29\%). El promedio de células TCD4 basal fue $284 \mathrm{cel} / \mathrm{mm}^{3}$, pero con valores extremos bajos de $11 \mathrm{cel} / \mathrm{mm}^{3}$.

E1 70\% de los pacientes tuvieron cumplimiento mayor al $80 \%$ de las citas, sin embargo sólo 13 pacientes (25\%) tuvieron carga viral indetectable a los seis meses de seguimiento; teniendo en cuenta la escala de Sacket, 12 pacientes (23\%) fueron adherentes a la terapia antirretroviral.

La Tabla 3 resume el perfil psicopatológico de la población, de resaltar 19 pacientes (37\%) presentaban características de trastorno disocial de la conducta antes de los 15 años, seis pacientes $(11 \%)$ cumplían criterios para personalidad

Tabla 2. Características clínicas y estado de inmunosupresión.

\begin{tabular}{|c|c|}
\hline Promedio IMC (Rango) & $22.5(17$ A 30$)$ \\
\hline \multicolumn{2}{|c|}{ Estado inmunológico } \\
\hline Promedio Conteo CD4 Basal & $284(11$ a 871$)$ \\
\hline Promedio Conteo CD4 a los seis meses & 295 (5 a 1334) \\
\hline Promedio Carga viral para VIH Basal & 107312 (0 a 2031050$)$ \\
\hline Promedio Carga viral para VIH 6 meses & 35205 (0 a 662930) \\
\hline \multicolumn{2}{|c|}{ Enfermedades concomitantes } \\
\hline Cardiovascular & $1(2 \%)$ \\
\hline Respiratorias & $2(3.9 \%)$ \\
\hline Diabetes Mellitus & $2(3.9 \%)$ \\
\hline Dislipidemia & $3(5.9 \%)$ \\
\hline Neurológicas & $2(3.9 \%)$ \\
\hline \multicolumn{2}{|c|}{ Oportunistas } \\
\hline Tuberculosis & $15(29 \%)$ \\
\hline Pneumocistis jiroveci & $3(5.9 \%)$ \\
\hline Toxoplasma gondi & $5(9.8 \%)$ \\
\hline Citomegalovirus (CMV) & $1(2 \%)$ \\
\hline Otros & $5(9.8 \%)$ \\
\hline Varias & $14(27 \%)$ \\
\hline
\end{tabular}


limítrofe y antisocial. En la Tabla 4 se describen los trastornos cognitivos acorde a escalas empleadas.

El conteo CD4 a los seis meses de seguimiento fue mayor en el grupo con adherencia a la terapia $\left(254 \mathrm{cel} / \mathrm{mm}^{3}\right.$ en no adherentes Vs $380 \mathrm{cel} / \mathrm{mm}^{3}$ adherentes), el promedio de carga viral a los seis meses de seguimiento en el grupo de no adherencia fue $44807 \mathrm{copias} / \mathrm{mm}^{3}$.

En el análisis bivariado encontramos que los factores asociados a abandono de la terapia antirretroviral son el

Tabla 3. Psicopatología

\begin{tabular}{|c|c|}
\hline & $\mathbf{n}(\%)$ \\
\hline Trastorno depresivo mayor & $6(11.8)$ \\
\hline Depresión mayor y melancolía & $4(7.8)$ \\
\hline Trastorno depresivo crónico tipo distimia & $6(11.8)$ \\
\hline \multicolumn{2}{|l|}{ Riesgo suicida } \\
\hline Leve & $6(11.8)$ \\
\hline Moderado & $2(3.9)$ \\
\hline Severo & $2(3.9)$ \\
\hline TAB crisis manía & $1(2)$ \\
\hline Trastorno psicótico síntomas esquizofrenia & $5(9.8)$ \\
\hline Trastorno ansiedad generalizado & $2(3.9)$ \\
\hline Trastorno ansiedad/crisis de pánico & $5(9.8)$ \\
\hline Trastorno de ansiedad/agorafobia & $2(3.9)$ \\
\hline Trastorno de ansiedad/fobia social & $1(2)$ \\
\hline Trastorno ansiedad /obsesivo compulsivo & $1(2)$ \\
\hline Ansiedad/estrés postraumático & $2(3.9)$ \\
\hline Bulimia & $1(2)$ \\
\hline Consumo abusivo de alcohol & $7(13.7)$ \\
\hline Dependencia de alcohol & $11(21.6)$ \\
\hline \multicolumn{2}{|l|}{ Consumo abusivo de psicoactivos } \\
\hline Nunca & $19(37)$ \\
\hline Cocaína & $1(2)$ \\
\hline Marihuana & $3(5.9)$ \\
\hline Alcohol & $7(13.7)$ \\
\hline Nicotina & $4(7.8)$ \\
\hline Otras & $8(15.7)$ \\
\hline Mas de 1 & $9(17.6)$ \\
\hline \multicolumn{2}{|l|}{ Trastorno personalidad } \\
\hline Esquizotípica & $1(2)$ \\
\hline Esquizoide & $1(2)$ \\
\hline Paranoide & $3(5.9)$ \\
\hline Histriónica & $3(5.9)$ \\
\hline Limítrofe & $6(11.8)$ \\
\hline Dependiente & $1(2)$ \\
\hline Obsesiva & $1(2)$ \\
\hline Antisocial & $6(11.8)$ \\
\hline Disocial de la conducta antes de los 15 años & $19(37.3)$ \\
\hline
\end{tabular}

estado civil soltero OR 4.57 (IC 95\% 1.13-18), no tener red de apoyo OR 1.33 (IC 95\% 1.13-1.57); tener un MMT mayor a 24 se encontró como factor protector OR 0.75 (IC 95\% 0.63-0.88), también no tener trastorno de ansiedad OR 0.7 (IC 95\% 0.58-0.86) Tabla 5.

Con estas variables se realizó regresión logística, ninguna demostró estar asociado a riesgo para abandono de la terapia (esto en asociación a bajo número de tamaño de muestra).

\section{Discusión}

En nuestra población con VIH/Sida la baja adherencia a la terapia antirretroviral puede explicar las tasas de inmunosupresión severa descrita y la gravedad con la que ingresan algunos de ellos a los servicios hospitalarios; aunque por el bajo tamaño de muestra no encontramos diferencias estadísticamente significativas en el análisis multivariado, los resultados bivariados nos sugieren posibles asociaciones a variables psicopatológicas y a la red de apoyo. La tasa de adherencia de nuestra población es baja comparada con otros estudios. Rodríguez et al en España, realizaron encuestas a 162 pacientes con enfermedad retroviral con TARGA y vigilancia activa en farmacia del número de tabletas dispensadas; la adherencia reportada por entrevista personal fue $63 \%$, pero al medirse por la recogida de la medicación fue tan solo $42 \%$ (19). En este estudio se encontró que el hecho de tener mayor número de comprimidos formulados diarios se asociaba a mayor riesgo de abandono a la terapia.

En el trabajo del Dr. Rodríguez Quesada (20), se evaluaron los factores que podrían afectar la adherencia al tratamiento antirretroviral en 211 pacientes en el hospital de Getafe, España; se utilizaron métodos indirectos para la medición de la misma (registro de dispensación de medicación en farmacia y el cuestionario de adherencia SMAQ),

Tabla 4. Trastornos cognitivos.

\begin{tabular}{|l|l|}
\hline \multicolumn{1}{|l|}{ Evaluación neurocognoscitiva } & n (\%) \\
\hline MMT* & $48(94)$ \\
\hline Mayor o igual a 24 & $3(5.9)$ \\
\hline Menor 24 & $25(49)$ \\
\hline MOCA** & $26(51)$ \\
\hline Mayor o igual a 26 & \\
\hline Menor a 26 & $31(60.8)$ \\
\hline Escala demencia SIDA & $20(39)$ \\
\hline Mayor o igual a 11 & \\
\hline Menor o igual a 10 & $16(31)$ \\
\hline SIDA déficit cognoscitivo & $17(33)$ \\
\hline Mayor 15 & $18(35)$ \\
\hline Entre 11 a 15 & \\
\hline Menor a 11 & \\
\hline$*$ Mini Mental Test * Montreal Cognitive assesment & \\
\hline
\end{tabular}


Tabla 5. Análisis univariado.

\begin{tabular}{|c|c|c|c|c|}
\hline Variable & Adherentes $\mathrm{N}=12$ & No adherentes $\mathrm{N}=39$ & $\mathbf{P}$ & OR (IC 95\%) \\
\hline Promedio de edad & 40.08 & 41 & 0.99 & \\
\hline CD4 6 meses & 444 & 261 & 0.036 & \\
\hline Sexo hombre/mujer & $10 / 2$ & $36 / 3$ & 0.36 & $2.4(0.35-6.3)$ \\
\hline Genero heterosexual/homosexual & $5 / 7$ & $20 / 19$ & 0.56 & $1.47(0.39-5.4)$ \\
\hline Estrato 0 y 1 /estrato 2 y 3 & $4 / 8$ & $8 / 31$ & 0.36 & $0.51(0.12-2.15)$ \\
\hline Soltero/casado & $6 / 6$ & $32 / 7$ & 0.026 & $4.57(1.13-18)$ \\
\hline Red de apoyo no/si & $0 / 12$ & $3 / 36$ & 0.322 & $1.33(1.13-1.57)$ \\
\hline Desempleado/empleado & $1 / 11$ & $11 / 28$ & 0.15 & $4.32(0.49-37)$ \\
\hline Ingreso entre 0 a $2 \mathrm{SMLV} /$ mayor a $2 \mathrm{SMLV}$ & $11 / 1$ & $37 / 2$ & 0.68 & $1.682(0.13-20)$ \\
\hline Consumo NO/SI & $6 / 6$ & $13 / 26$ & 0.296 & $0.5(0.13-1.85)$ \\
\hline DX menor 2 años/mayor 2 años & $5 / 7$ & $18 / 21$ & 0.78 & $1.2(0.32-4.44)$ \\
\hline Depresión NO/SI & $9 / 3$ & $32 / 7$ & 0.59 & $1.52(0.32-7.11)$ \\
\hline Ansiedad NO/SI & $12 / 0$ & $29 / 10$ & 0.05 & $0.70(0.58-0.86)$ \\
\hline Trastorno personalidad NO/SI & $7 / 5$ & $26 / 13$ & 0.59 & $1.42(0.37-5.39)$ \\
\hline Riesgo suicida NO/SI & $11 / 1$ & $30 / 9$ & 0.26 & $0.3(0.034-2.6)$ \\
\hline Trastorno disocial conducta NO/SI & $9 / 3$ & $23 / 16$ & 0.31 & $0.47(0.11-2.05)$ \\
\hline MMT mayor $24 /$ menor 24 & $12 / 0$ & $36 / 3$ & 0.322 & $0.75(0.63-0.88)$ \\
\hline MOCA mayor a $26 /$ menor a 26 & $7 / 5$ & $18 / 21$ & 0.46 & $0.61(0.16-2.26)$ \\
\hline Escala demencia sida mayor 11 /menor 11 & $8 / 4$ & $23 / 16$ & 0.63 & $0.71(0.18-2.7)$ \\
\hline Déficit cognitivo mayor $15 /$ menor 15 & $3 / 9$ & $13 / 26$ & 0.58 & $1.5(0.34-6.5)$ \\
\hline Psicopatología NO/SI & $5 / 7$ & $13 / 26$ & 0.59 & $0.7(0.18-2.6)$ \\
\hline Consumo psicoactivos NO/SI & $6 / 6$ & $13 / 26$ & 0.29 & $0.5(0.13-1.85)$ \\
\hline
\end{tabular}

con unos niveles de adherencia de $65.8 \%$ con los registros de farmacia, y de $53 \%$ con el formulario. En este grupo de pacientes la edad resultó ser un factor importante para la adherencia; el hecho de haber adquirido el virus por el uso de drogas intravenosas, el consumo de alcohol, la confección con el virus de hepatitis $\mathrm{C}$ y el tiempo desde el diagnóstico se asociaron en forma negativa con la adherencia a la terapia. Como factores protectores se encontraron el nivel educativo y el tipo de medicación utilizada (regímenes que incluyeran inhibidores de transcriptasa reversa no nucleotídicos). La población estudiada maneja frecuencias altas de psicopatología asociada (incluidos los trastornos cognitivos), que en conjunto con la farmacodependencia, la vulnerabilidad socioeconómica y la baja red de apoyo se convierten en factores que muy probablemente se asocien a abandono a la terapia en este grupo de pacientes.

Los resultados encontrados hacen pensar que al intervenir estas variables, a través de un manejo interdisciplinario, se podría impactar en forma positiva los desenlaces de la enfermedad; Navarro et al. (7) evaluaron la eficacia de un programa de cumplimiento en una cohorte de pacientes multitratados y poco adherentes; el programa incluyó un equipo multidisciplinar con una enfermera que se especializó en la intervención conductual, asesoramiento sobre abuso de sustancias, y la entrevista motivacional, así como un trabajador social responsable de la derivación de pacientes a los centros de salud locales. La eficacia se evaluó como porcentaje de pacientes con carga viral $<50$ copias $/ \mathrm{mL}$ a la semana 48. Inicialmente el $30 \%$ de los pacientes tenían adherencia acorde al cuestionario SMAQ. Luego de la intervención $48.1 \%$ de los pacientes tenían cargas virales indetectables y la adherencia reportada fue $>90 \%$.

En nuestra población, la presencia de infecciones oportunistas como la tuberculosis es reflejo de la baja adherencia a los antirretrovirales; el estado de inmunosupresión severa incrementa además de la mortalidad, el riesgo de síndrome de reconstitución inmunológica; la polifarmacia afecta en forma negativa la adherencia a cualquier tratamiento médico y esto es lo que con frecuencia se tiene cuando se está cursando con una infección de esta categoría o cualquier otra oportunista, hecho descrito por Cantudo-Cuenca et al. (21); aunque estas variable no fue incluida en la cohorte se considerarán para futuros seguimientos, así como la baja adherencia secundaria a eventos adversos de la medicación.

Como limitaciones encontramos una muestra reducida de pacientes que influye en el poder estadístico para identificación de diferencias; las escalas para la detección de trastornos neurocognitivos no están validadas en nuestro 
país; sin embargo, este es un primer esfuerzo en la descripción de las mismas, ya que es fundamental la identificación de dichos trastornos que pueden impactar directamente en la adherencia a la medicación. La mayoría de los pacientes procedían de la consulta externa y consideramos puede haber una gran diferencia en relación al paciente hospitalizado; lo anterior lleva al planteamiento de nuevas preguntas que se deberán responder con nuevos diseños.

Este es el primer estudio en nuestro medio en relación a abandono a terapia antirretroviral, donde logramos mostrar que podría existir relación entre el consumo de sustancias y el abandono a la terapia, así como influencia directa del soporte social para el mantenimiento de la misma. Estos factores son potencialmente modificables y deben conducir a políticas de salud destinadas a vencer las barreras que garanticen continuidad en las terapias.

\section{Conclusión}

La pobre red de apoyo y la psicopatología (trastorno de ansiedad y déficit cognitivo) están asociados a mayor riesgo de abandono de la terapia antirretroviral de alta efectividad en nuestra población. Si bien no se logró demostrar asociación con el consumo de sustancias psicoactivas, existe una alta frecuencia de esta patología y no desconocemos el riesgo de los pacientes para la no adherencia y la alta vulnerabilidad.

\section{Conflictos de interés}

Aclaramos para el desarrollo de esta investigación no tenemos conflictos de interés y ésta no fue patrocinada por agentes externos ni contó con ningún tipo de presupuesto diferente a los estipulado en las actividades propias del servicio.

\section{Referencias}

1. Ministerio de Protección social / Sociedad Colombiana de Infectología ACIN. Guía de práctica clínica basada en la evidencia científica para la atención de la infección por VIH/Sida en adolescentes (con 13 años de edad o más) y adultos. Disponible en: http://www.acin.org/acin/new/Portals/0/Templates/GPC_Comple_ VIHADULTOS_web.pdf

2. Informe ONUSIDA Tratamiento 2015. Disponible en: http://www.unaids.org/ sites/default/files/sub_landing/files/JC2484_treatment-2015_es.pdf

3. Organización panamericana de la Salud. Protocolo para implementación de indicadores de alerta temprana para la farmacorresistencia del VIH en Colombia en adolescentes y adultos 2014. Disponible en: http://www.dadiscartagena.gov. co/images/docs/saludpublica/vigilancia/d2016/ppi_indicadores_at_vih.pdf

4. Viswanathan S, Detels R, Mehta SH, Macatangay BJ, Kirk GD, Jacobson LP. Level of Adherence and HIV RNA Suppression in the Current Era of Highly Active Antiretroviral Therapy (HAART). AIDS Behav 2015; 19(4).

5. Bangsberg DR,Hecht FM,Charlebois ED,Zolopa AR,Holodniy M,Sheiner L,Bamberger JD,Chesney MA,Moss A. Adherence to protease inhibitors,HIV-1 viral load, and development of drug resistance in anindigentpopulation. AIDS 2000; 14(4): 357-66

6. Silva J.A, Doruado, De Brito AM, Lima de Silva C.A. Factors associated with non-adherence to antiretroviral therapy in adults with AIDS in the first six months of treatment in Salvador, Bahia State, Brazil. Cad. Saúde Pública 2015;31(6):1-11.

7. Navarro J, Pérez M, Curran A, Burgos J,Feijoo M, RN, Torrella A et al. Impact of an adherence program to antiretroviral treatment on virologic response in a cohort of multitreated and poorly adherent HIV-infected patients in Spain. AIDS Patient Care STDS. 2014; 28(10): 537-42.

8. Sackotor, Ned C, WongMatthew; et al. "The International HIV Dementia Scale: a new rapid screening test for HIV dementia." AIDS 2005; 19(13): 1367-74.

9. Michael F, Gibbon M, Spitzer R. Entrevista Clínica estructurada para los Trastornos de Personalidad del eje II del DSM IV. Editorial Masson. Adaptación española, 1999.

10. Michael F, Gibbon M, Spitzer R. Entrevista Clínica estructurada para los Trastornos de Personalidad del eje II del DSM IV. Editorial Masson. Adaptación española, 1999.

11. Ferramdo L, Bobes J, Gilbert J. Mini International Neuropsychiatric Interview. Versión en Español 5.0.0. International DSM-IV Instituto IAP-Madrid - España. Enero 2000: 1-26

12. Sheehan D, Janavs L., Baker R,Harnett-Sheehan K., Knapp E, SheehanY, et al. Mini International Neuropsychiatric Interview Hôpital de la Salpétrière, Paris. University of South Florida, Tampa. Julio 2006:1 -27

13. Folstein MF, Folstein SE, PR MF, McHugh PR. "Mini-mentalstate": a practical method for grading the cognitive state of patients for the clinician. J Psychiatr Res 1975;12(3):189-98.

14. Nasreddine ZS, Phillips NA, Be'dirian V, et al. The Montreal Cognitive Assessment, MoCA: a brief screening tool for mild cognitive impairment. J Am Geriat Soc. 2005; 53: 695-99.

15. Selnes A.; Julie A.; McArthur J. HIV Dementia Scale: A Rapid Screening Test; J Acquir Immune Defic Syndr Hum Retrovirol. 1995; 8(3): 273-78.

16. Custodio N, Escobar J, Altamirano J. Demencia asociada a infección por virus de inmunodeficiencia humana tipo 1. An Fac Med Lima 2006; 67(3): 243-54

17. Berghuis J, Uldall $\mathbf{k}$, Lalonde B. Validity of Two Scales in identifying HIVAssociated Dement. J Adq Inm Def Synd 1999; 21: 134-40

18. Panel de expertos de SPNS, SEFH y GESIDA. Mejorar la adherencia al tratamiento antirretroviral. Recomendaciones de la SPNS/SEFH/GESIDA. Farm Hosp. 2008;32(6):349-57

19. Rodríguez G, Iranzu Aperte M, Berrocal J, Gómez M et al. Adherencia a tratamiento antirretroviral: repercusión del número de tomas diarias. Rev Cubana Farm 2009; 43(1).

20. Rodriguez $\mathbf{P}$. Estudio de factores de riesgo influyentes en el adherencia al tratamiento antirretroviral y desarrollo de un modelo predictivo en pacientes VIH positivos. Memoria para optar al grado de Doctor, Universidad Complutense de Madrid, Facultad de farmacia. 2016. Consultado en: http://eprints.ucm.es/36230/

21. Cantudo-Cuenca MR, Jiménez-Galán R, Almeida-Gonzalez CV, MorilloVerdugo R. Concurrent use of comedications reduces adherence to antiretroviral therapy among HIV-infected patients. J Manag Care Pharm 2014; 20(8): 844-50. 